University of Wollongong

Research Online

Faculty of Engineering and Information

Faculty of Engineering and Information

Sciences - Papers: Part A

Sciences

$1-1-2012$

Multiuser diversity with capture in OFDMA systems with clustered feedback

Hang Li

University Of Western Australia

Qinghua Guo

University of Wollongong, qguo@uow.edu.au

Licai Fang

University Of Western Australia

Defeng (David) Huang

University Of Western Australia, david.huang@uwa.edu.au

Follow this and additional works at: https://ro.uow.edu.au/eispapers

Part of the Engineering Commons, and the Science and Technology Studies Commons

Research Online is the open access institutional repository for the University of Wollongong. For further information contact the UOW Library: research-pubs@uow.edu.au 


\title{
Multiuser diversity with capture in OFDMA systems with clustered feedback
}

\begin{abstract}
In this paper, we propose a cluster-based feedback protocol for multiuser orthogonal frequency-division multipleaccess (OFDMA) systems, where the capture effect is employed in each cluster to reduce the enormous system overhead induced by polling channel-state information. Then, we analyze the system throughput performance of the proposed feedback protocol. Compared with the full feedback scheme in the literature, numerical simulations show that the proposed protocol with clustered feedback in OFDMA systems achieves higher system throughput by employing the capture effect with limited feedback overhead, when the number of users is reasonably large. Moreover, compared with our previous protocol of Multiuser Diversity with Capture, the proposed one additionally exploits multiuser diversity in the frequency domain, thereby achieving better throughput performance as shown by the simulation results.
\end{abstract}

\section{Keywords}

systems, feedback, ofdma, clustered, capture, diversity, multiuser

Disciplines

Engineering | Science and Technology Studies

\section{Publication Details}

H. Li, Q. Guo, L. Fang \& D. Huang, "Multiuser diversity with capture in OFDMA systems with clustered feedback," in International Conference on Wireless Communications and Signal Processing, 2012, pp. 1-5. 


\title{
Multiuser Diversity with Capture in OFDMA Systems with Clustered Feedback
}

\author{
Hang $\mathrm{Li}^{*}$, Qinghua Guo*†, Licai Fang* and Defeng (David) Huang* \\ *School of Electrical, Electronic and Computer Engineering, \\ The University of Western Australia, 35 Stirling Highway, Crawley, WA 6009, Australia \\ ${ }^{\dagger}$ School of Electrical, Computer and Telecommunications Engineering, \\ University of Wollongong, NSW 2522, Australia \\ Email: hangli@ee.uwa.edu.au, qinghua_guo@uow.edu.au, licaifang@gmail.com, david.huang@uwa.edu.au
}

\begin{abstract}
In this paper, we propose a cluster-based feedback protocol for multiuser orthogonal frequency-division multipleaccess (OFDMA) systems, where the capture effect is employed in each cluster to reduce the enormous system overhead induced by polling channel-state information. Then, we analyze the system throughput performance of the proposed feedback protocol. Compared with the full feedback scheme in the literature, numerical simulations show that the proposed protocol with clustered feedback in OFDMA systems achieves higher system throughput by employing the capture effect with limited feedback overhead, when the number of users is reasonably large. Moreover, compared with our previous protocol of Multiuser Diversity with Capture, the proposed one additionally exploits multiuser diversity in the frequency domain, thereby achieving better throughput performance as shown by the simulation results.

Index Terms-Capture effect, clustered feedback, multiuser diversity, orthogonal frequency-division multiple-access (OFDMA).
\end{abstract}

\section{INTRODUCTION}

Multiuser diversity can be exploited over time-varying independent fading channels among different users at different times to significantly increase the system throughput of a wireless network, where the channel is always scheduled to the user with the best channel [1], [2]. In general, multiuser diversity gain increases as the number of users increases. However, the amount of feedback overhead also increases with the number of users [3], and the feedback overhead induced by exploiting multiuser diversity may overshadow the multiuser diversity gain. In multiuser orthogonal frequencydivision multiple-access (OFDMA) downlink transmission, clusters [i.e., a number of adjacent orthogonal frequencydivision multiplexing (OFDM) subcarriers] are used as basic units for channel allocation [4], [5]. Multiuser diversity can independently be achieved in each cluster [6]. However, in this scenario, the amount of feedback overhead increases with not only the number of users, but also the number of clusters. Therefore, reducing feedback overhead becomes more significant for multiuser OFDMA systems.

To handle the overhead issue, a one-bit-per-subcarrier (or cluster) scheme was proposed in [7], [8] for OFDM systems, where only one-bit information per subcarrier (or cluster) that indicates whether the user's subcarrier (or cluster) channel gain

This work was supported by Australian Research Council's Discovery Projects DP0877616 and DP1093000, and DECRA Grant DE120101266. is above a threshold, is fed back to the base station (BS) for subchannel allocation. In [6] and [9], the best- $M$ feedback strategy was presented, where the indices and channel gains of the $M$ subcarriers with the best channel are fed back to the BS. Although the amount of feedback is reduced in [6]-[9], the overhead required still linearly increases with the number of users.

In wireless communications, the capture effect is the ability of a radio receiver to receive a signal from one transmitter despite interference from one or more other transmitters, and it is particularly prominent when the receiver is capable of working in the low signal-to-interference-plus-noise ratio region. Contention-based feedback protocol, namely Multiuser Diversity with Capture in [10], was proposed to explicitly employ the capture effect to obviate the overhead problem in conventional OFDM systems, where all users share the same feedback slot. By employing the capture effect, higher throughput [11] can be achieved compared with the case that capture is not considered. However, in [10] and [11], multiuser diversity is solely considered in the time domain, and can not be achieved in the frequency domain.

In this paper, we propose a cluster-based feedback protocol to reduce the system overhead by utilizing the capture effect for multiuser OFDMA systems. Only the users with received signal-to-noise ratios (SNRs) on a cluster above a threshold send a feedback message in the corresponding cluster. The capture effect is then exploited at the BS to identify the user with the largest SNR and therefore the best channel. We analyze the system throughput performance of the proposed feedback protocol over frequency selective fading channels with uniform power delay profile (UPDP). Both numerical and simulation results show that by explicitly using the capture effect to reduce system overhead, the proposed feedback protocol achieves much better throughput performance than that of the full feedback scheme [12], especially when the radio receiver has reasonably good capture properties or when the number of users is reasonably large. Our previous protocol [10] is a special case of the proposed one in this paper, i.e., the number of clusters is 1 . As additional multiuser diversity can be exploited in the frequency domain, the proposed feedback protocol outperforms the previous one in system throughput with the increase of the number of clusters. 
The rest of this paper is organized as follows. In Section II, we describe the system model, which includes downlink signal model, the proposed feedback protocol and capture model. Then, the throughput performance analysis of the proposed feedback protocol is given in Section III. In Section IV, we present the numerical and simulation results. Finally, Section $\mathrm{V}$ concludes this paper.

\section{SYSTEM MODEL}

\section{A. Downlink Signal Model}

We consider the downlink transmission with one BS and $K$ users in a wireless network, where the total transmission power at the BS is a constant and the uplink and downlink channels are reciprocal. The $N$ OFDM subcarriers are grouped into $M$ adjacent clusters, each with $P=N / M$ subcarriers, and each cluster spans over a number of OFDM symbols in the time domain. The cluster can not only be used as the basic physical unit for multiuser downlink data transmission, but also as feedback channel to reduce the amount of overhead from users compared to the per-subcarrier based scheme. We assume that all active users are ideally synchronized to the BS, and the data for active users is infinitely backlogged; therefore, the queueing dynamics is not considered. The BS transmits data packets at each cluster to only one user in every downlink frame.

We assume that the channel between the BS and each user is frequency selective fading, block-wise time invariant for each frame and changes independently from frame to frame. The discrete-time baseband equivalent channel between the BS and the $k$ th user is denoted by the following vector:

$$
\mathbf{h}_{k}=\left[h_{k}(0), h_{k}(1), \ldots, h_{k}(L-1)\right]^{T}
$$

where $(\cdot)^{T}$ in the superscript denotes transpose, $h_{k}(l)$ denotes the $k$ th user's channel impulse response for the $l$ th tap, and $L$ is the maximum number of channel taps for all users. By taking the discrete Fourier transform, the channel frequency response $H_{k}[p, m]$ for the $k$ th user at the $p$ th subcarrier of the $m$ th cluster is obtained by

$$
H_{k}[p, m]=\sum_{l=0}^{L-1} h_{k}(l) e^{-j 2 \pi[(m-1) P+p-1] l / N}
$$

where $p=1,2, \ldots, P$ and $m=1,2, \ldots, M$. Therefore, the received signal $Y_{k}[p, m]$ of the $k$ th user at the $p$ th subcarrier of the $m$ th cluster is given by

$$
Y_{k}[p, m]=H_{k}[p, m] S_{k}[p, m]+Z_{k}[p, m]
$$

where $S_{k}[p, m]$ and $Z_{k}[p, m]$ are the transmitted signal at the BS and the additive white circularly symmetric complex Gaussian noise at the user side for the $k$ th user at the $p$ th subcarrier of the $m$ th cluster, respectively.

\section{B. Proposed Feedback Protocol Description}

Fig. 1 shows the framing structure of the proposed feedback protocol (Note it is an extension of our work in [10]). The BS first informs all active users to respond by a broadcast message,

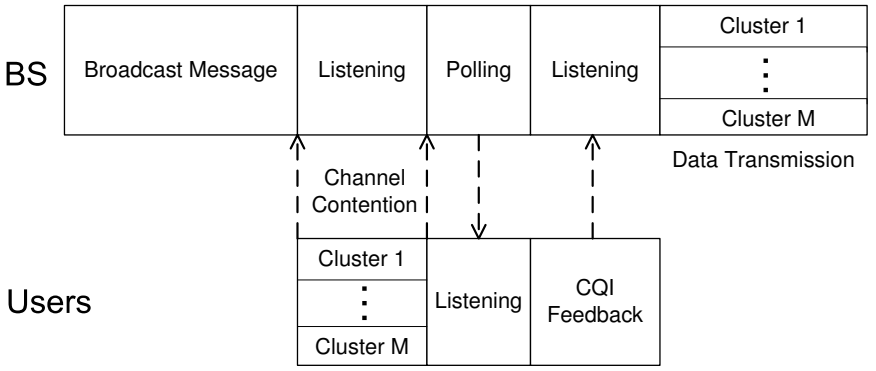

Fig. 1. The framing structure of the feedback protocol with clustered feedback.

which includes system configuration information (e.g., the values of the feedback thresholds ${ }^{1}$ for all clusters). Using the broadcast message, all users synchronize with the BS and estimate their received SNRs on all clusters. Then, only the users whose SNRs on a cluster are greater than their feedback thresholds, feed back a packet carrying their user identity information in the corresponding cluster.

Based on the received packets in every cluster, the BS seeks to identify the user with the greatest received SNR on every cluster and allocates it the channel so that it may perform data transmission. However, the BS will only be able to correctly identify the user with the greatest received SNR on a cluster if (i) there is exactly one user's feedback, or (ii) the strongest signal can be captured from multiple users' feedback. When the user with the greatest received SNR is identified on a cluster, that user is allocated the channel to transmit data in the corresponding cluster. Otherwise, the BS has not obtained any information about which user has the best channel and it allocates the channel to a randomly chosen user. By randomly chosen, we mean that the index of the user is a uniformly distributed random variable from 1 to $K$. When the BS has decided which users will be allocated the channels for all clusters, the BS polls and requests them to report their channel quality information (CQI, e.g., the received SNR or alternative channel quality indicator) on the corresponding clusters. Finally, using the CQI feedback, the $\mathrm{BS}$ selects an appropriate transmission rate which is assumed to be the downlink channel capacity for each cluster.

\section{Capture Model}

The capture effect is a complex phenomenon that depends on the specific implementation of the receiver, and some capture models have been proposed to characterize it [13], [14]. In this paper, we employ the capture ratio model [13] in the uplink cluster-based feedback. The signal of a user is captured (i.e., decoded correctly by the receiver) if and only if at the BS, the ratio of the received signal power from this user to the total interference power from other users is greater

${ }^{1}$ Given the channel gain distribution on each cluster, the feedback thresholds can be pre-computed and then used at the BS. The channel gain distribution can also be estimated at the BS dynamically by collecting the uplink feedback information. 
than a capture ratio $z$, where the value of $z$ depends on the receiver characteristics and system requirements.

Let $n>1$ be the number of feedback users in a feedback channel (e.g., one cluster), whose indices and the corresponding received SNRs are $v_{1}, v_{2}, \ldots, v_{n}$ and $\Gamma_{v_{1}}, \Gamma_{v_{2}}, \ldots, \Gamma_{v_{n}}$, respectively. Without loss of generality, let $v_{1}$ be the index of the user with the greatest received SNR, and the capture ratio model can be expressed as follows:

$$
\Gamma_{v_{1}}>z \sum_{i=2}^{n} \Gamma_{v_{i}}
$$

When $0<z<1$, multiple users may be captured if (4) is satisfied. However, this is out of the scope of this paper, and we consider the case that only one user may be captured (i.e., $z \geq 1$ ).

\section{Performance Analysis of the Proposed FEEDBACK PROTOCOL}

\section{A. The Average Sum Rate}

We define the instantaneous channel gain for the $k$ th user on the $m$ th cluster as follows:

$$
\alpha_{k}[m]=\frac{1}{P} \sum_{p=1}^{P}\left|H_{k}[p, m]\right|^{2} .
$$

Therefore, the received SNR for the $k$ th user on the $m$ th cluster is given by

$$
\Gamma_{k}[m]=\rho \alpha_{k}[m]
$$

where we assume that the transmit power allocated to all subcarriers is the same for all users, and $\rho$ is the ratio of the transmit power to the noise power at the receiver.

Let $R_{k}[m]$ denotes the event that $k$ of the $K$ users in the network feed back a message in the $m$ th cluster and let $G[m]$ be a random variable that represents the data rate in the $m$ th cluster. Applying the law of total expectation, the average sum rate $(\mathrm{bps} / \mathrm{Hz})$ is given by

$$
E[G]=\frac{1}{M} \sum_{m=1}^{M} \sum_{k=0}^{K} E\left[G[m] \mid R_{k}[m]\right] P\left(R_{k}[m]\right) .
$$

Let $C[m]$ denote the event that the BS is able to correctly identify the user with the best channel during the channel contention of the $m$ th cluster. Applying the law of total expectation again, we have

$$
\begin{aligned}
& E\left[G[m] \mid R_{k}[m]\right] \\
= & E\left[G[m] \mid C[m], R_{k}[m]\right] P\left(C[m] \mid R_{k}[m]\right) \\
& +E\left[G[m] \mid \bar{C}[m], R_{k}[m]\right] P\left(\bar{C}[m] \mid R_{k}[m]\right)
\end{aligned}
$$

where $\bar{C}[m]$ represents the set complement of $C[m]$.

We assume that the fadings that the users experience are independently and identically distributed (i.i.d.), hence the $\Gamma_{k}[m] \mathrm{s}$ for all users on the $m$ th cluster are i.i.d. random variables, each with probability density function (pdf) $f_{\Gamma[m]}(\cdot)$ and cumulative distribution function (cdf) $F_{\Gamma[m]}(\cdot)$. Let the indices of the users that do not feed back a message in one cluster to the BS be $u_{1}, u_{2}, \ldots, u_{K-k}$ and the indices of the users that feed back a message in one cluster to the BS be $v_{1}, v_{2}, \ldots, v_{k}$. Since the $\Gamma_{i}[m] \mathrm{s}$ for all users are i.i.d., it follows that the $\Gamma_{u_{j}}[m] s$ are i.i.d. and that the $\Gamma_{v_{n}}[m] s$ are also i.i.d.. Denote the pdfs of $\Gamma_{u_{j}}[m] \mathrm{s}$ and $\Gamma_{v_{n}}[m] \mathrm{s}$ by $f_{U[m]}(\cdot)$ and $f_{V[m]}(\cdot)$, and their cdfs by $F_{U[m]}(\cdot)$ and $F_{V[m]}(\cdot)$, respectively. It is shown that

$$
\begin{gathered}
f_{U[m]}(x)=\frac{f_{\Gamma[m]}(x)}{F_{\Gamma[m]}(\eta[m])}, \quad 0 \leq x<\eta[m], \\
F_{U[m]}(x)=\frac{F_{\Gamma[m]}(x)}{F_{\Gamma[m]}(\eta[m])}, \quad 0 \leq x<\eta[m], \\
f_{V[m]}(x)=\frac{f_{\Gamma[m]}(x)}{1-F_{\Gamma[m]}(\eta[m])}, \quad x \geq \eta[m], \\
F_{V[m]}(x)=\frac{F_{\Gamma[m]}(x)-F_{\Gamma[m]}(\eta[m])}{1-F_{\Gamma[m]}(\eta[m])}, \quad x \geq \eta[m]
\end{gathered}
$$

where $\eta[m]$ is the feedback threshold on the $m$ th cluster.

Further, let the pdf and cdf of the sum of $k$ independent random variables with pdf $f_{V[m]}(\cdot)$ be $f_{V[m], k}(\cdot)$ and $F_{V[m], k}(\cdot)$, respectively. It follows that $f_{V[m], k}(\cdot)$ is the $k$-fold convolution of $f_{V[m]}(\cdot)$. For convenience, we define $f_{V[m], 0}(t)=\delta(t)$, where $\delta(t)$ is the Dirac delta function. Let $g(x)$ be the transmitted data bit rate per Hertz given the received SNR $x$.

Without loss of generality, we focus on the analysis of the sum rate for the $m$ th cluster in the following equations. For simplicity of notation, we ignore the cluster index $[\mathrm{m}]$. Using similar methods as shown from (8)-(15) in [10], we can obtain the following equations,

$$
\begin{array}{r}
P\left(R_{k}\right)=\left(\begin{array}{c}
K \\
k
\end{array}\right)\left(1-F_{\Gamma}(\eta)\right)^{k} F_{\Gamma}(\eta)^{K-k}, \\
P\left(C \mid R_{k}\right)=\left\{\begin{array}{lr}
0, & k=0 \\
1, & k=1 \\
k \int_{(k-1) \eta}^{\infty}\left(1-F_{V}(z x)\right) f_{V, k-1}(x) d x, & k \geq 2
\end{array}\right. \\
P\left(\bar{C} \mid R_{k}\right)=1-P\left(C \mid R_{k}\right), \\
E\left[G \mid C, R_{k}\right]= \begin{cases}0, & (14) \\
\int_{\eta}^{\infty} g(x) f_{V}(x) d x, & k=1 \\
\frac{k}{P\left(C \mid R_{k}\right)} a_{k}, & k \geq 2\end{cases}
\end{array}
$$

where

$$
a_{k}=\int_{z(k-1) \eta}^{\infty} g(x) F_{V, k-1}\left(\frac{x}{z}\right) f_{V}(x) d x
$$

and

$$
E\left[G \mid \bar{C}, R_{k}\right]=\left\{\begin{array}{lr}
\int_{0}^{\eta} g(x) f_{U}(x) d x, & k=0 \\
0, & k=1 \\
\frac{K-k}{K} E\left[G \mid \bar{C}, R_{0}\right]+\frac{k}{K P\left(\bar{C} \mid R_{k}\right)} b_{k}, & k \geq 2 \\
(18)
\end{array}\right.
$$

where

$$
b_{k}=E\left[G \mid C, R_{1}\right]-a_{k}-(k-1) c_{k},
$$


TABLE I

$$
\begin{aligned}
& c_{k}= \\
& \int_{\eta}^{\infty} \int_{(k-2) \eta}^{\infty} g(x) f_{V}(x)\left(1-F_{V}(z(x+y))\right) f_{V, k-2}(y) d y d x .
\end{aligned}
$$

For a practical system, we assume that the physical layer (PHY) is capable of rate adaptation and selects the highest feasible data rate given the CQI of the selected user. Specifically,

$$
g(x)= \begin{cases}0, & x<x_{1} \\ g_{j}, & x_{j} \leq x<x_{j+1}, \quad \forall 0<j<J \\ g_{J}, & x \geq x_{J}\end{cases}
$$

where $g_{j}$ is the average data bit rate per Hertz for PHY mode $j, J$ is the number of PHY modes and the mode threshold $x_{j}$ is the minimum SNR required to transmit data at PHY mode $j$.

\section{B. System Throughput}

Since wireless channels are time-varying and the size of the data packet can not be too large, the overhead induced by the control packets should be considered when we calculate the system throughput. We define $t_{\text {data }}$ and $t_{\text {overhead }}$ to be the packet transmission duration for the data and the overhead of all control packets, respectively. Therefore, the system throughput is defined as

$$
T=\frac{t_{\text {data }} \cdot E[G] \cdot B}{t_{\text {data }}+t_{\text {overhead }}}=\frac{a \cdot E[G] \cdot B}{a+Q}
$$

where $B$ is the channel bandwidth, and we assume that the size of all the control packets is the same, $a$ is the ratio of the size of the data packet to that of one control packet and $Q$ is the number of control packets.

\section{Frequency Selective Fading Channels with Uniform Power Delay Profile}

We assume that all users' channels follow i.i.d. frequency selective fadings with UPDP, and each channel tap follows complex Gaussian distribution with zero mean and variance $1 / L$. Let $\mathbf{F}_{p, m}=$ $\left[1, e^{-j 2 \pi[(m-1) P+p-1] / N}, \ldots, e^{-j 2 \pi[(m-1) P+p-1](L-1) / N}\right]^{T}$, and we have [15]

$$
\Gamma_{k}[m]=\frac{\rho}{P} \sum_{p=1}^{P}\left|\mathbf{F}_{p, m}^{H} \mathbf{h}_{k}\right|^{2}=\mathbf{h}_{k}^{H} \boldsymbol{\Sigma}_{m} \mathbf{h}_{k}
$$

where $\boldsymbol{\Sigma}_{m}=\frac{\rho}{P} \sum_{p=1}^{P} \mathbf{F}_{p, m} \mathbf{F}_{p, m}^{H}$. By performing an eigendecomposition, we have $\boldsymbol{\Sigma}_{m}=\mathbf{V}_{m}^{H} \boldsymbol{\Lambda}_{m} \mathbf{V}_{m}$, and $\boldsymbol{\Lambda}_{m}=$ $\operatorname{diag}\left\{\lambda_{m, 1}, \ldots, \lambda_{m, L}\right\}$, where $\operatorname{diag}(\cdot)$ denotes a diagonal matrix, with the entries in the main diagonal given by $(\cdot) . \mathbf{V}_{m} \mathbf{h}_{k}$ also follows i.i.d. complex Gaussian distribution with zero mean and variance $1 / L$, hence the pdf of $\Gamma[\mathrm{m}] \mathrm{s}$ for all users can be written as [15]

$$
f_{\Gamma[m]}(x)=\sum_{l=1}^{L} A_{m, l} \lambda_{m, l}^{-1} e^{-\lambda_{m, l}^{-1} x} I\left\{\lambda_{m, l} \neq 0\right\}
$$

MODULATION AND CODING SCHEMES

\begin{tabular}{|c|c|c|c|c|}
\hline $\begin{array}{c}\text { MCS level } \\
j\end{array}$ & $\begin{array}{c}\text { Modulation } \\
\text { schemes }\end{array}$ & $\begin{array}{c}\text { Coding } \\
\text { rate }\end{array}$ & $\begin{array}{c}\text { Data rate } \\
g_{j}(\mathrm{bps} / \mathrm{Hz})\end{array}$ & $\begin{array}{c}\text { MCS level } \\
\text { threshold, } x_{j}(\mathrm{~dB})\end{array}$ \\
\hline \hline 1 & QPSK & $1 / 12$ & 0.16 & -5.6 \\
2 & QPSK & $1 / 8$ & 0.25 & -3.8 \\
3 & QPSK & $1 / 4$ & 0.5 & -1.4 \\
4 & QPSK & $1 / 2$ & 1.0 & 2.1 \\
5 & QPSK & $3 / 4$ & 1.5 & 6.6 \\
6 & 16-QAM & $1 / 2$ & 2.0 & 7.2 \\
7 & 16-QAM & $3 / 4$ & 3.0 & 12.2 \\
8 & 64-QAM & $1 / 2$ & 3.0 & 12.5 \\
9 & 64-QAM & $2 / 3$ & 4.0 & 16.3 \\
10 & 64-QAM & $3 / 4$ & 4.5 & 18.6 \\
11 & 64-QAM & $5 / 6$ & 5.0 & 22.8 \\
\hline
\end{tabular}

where $A_{m, l}=\prod_{q=1, q \neq l}^{L} \frac{\lambda_{m, q}^{-1}}{\lambda_{m, q}^{-1}-\lambda_{m, l}^{-1}} I\left\{\lambda_{m, q} \neq 0, \lambda_{m, l} \neq 0\right\}$ [16], and $I\{\cdot\}$ denotes the indicator function. Therefore, the $F_{\Gamma[m]}(x)$ can be obtained as

$$
F_{\Gamma[m]}(x)=\sum_{l=1}^{L} A_{m, l}\left(1-e^{-\lambda_{m, l}^{-1} x}\right) I\left\{\lambda_{m, l} \neq 0\right\} .
$$

Note that, by substituting (21), (24) and (25) into (9)(20), we can obtain the average sum rate $E[G]$ and therefore the system throughput (22) over frequency selective fading channels with UPDP.

\section{Numerical AND Simulation Results}

In this section, we present the numerical and simulation results of the system throughput for the proposed feedback protocol with $\rho=12 \mathrm{~dB}$ over a 4-tap channel with UPDP. In the simulations, we employ $N=128, B=1.25 \mathrm{MHz}$ and $J=11$ modulation and coding schemes (MCSs) levels based on the IEEE 802.16e [17], and use the corresponding thresholds $\left\{x_{j}\right\}_{j=1}^{J}$ in [18] with the target packet error rate of $\varepsilon=1 \%$ through the link level simulation, as shown in Table I. Also, Table I gives the average data rate $g_{j}$ with the modulation and coding rate. The ratio of the size of the data packet to that of one control packet, $a$, is fixed to be 10 , and as indicated in Fig. 1, the number of the control packets, $Q$, is 4 . All the simulation results are averaged over 30000 frames.

Fig. 2 shows the throughput performances for the proposed feedback protocol and the full feedback scheme versus the number of users, where the number of clusters is fixed to be 4. For the full feedback scheme, all active users' overhead that is carried in the control packet is fed back one by one; thus, the number of the control packets required is $K+1$. The numerical results for the system throughput are obtained using order statistic analysis. For the proposed feedback protocol, the feedback thresholds have been numerically optimized to the nearest $0.1 \mathrm{~dB}$ so that the system throughput is maximized for each value of $K$ and $z$. As shown in Fig. 2, with the increase of the number of users, the overhead incurred from polling users overshadows potential multiuser diversity gain for the full feedback scheme. The proposed feedback protocol 


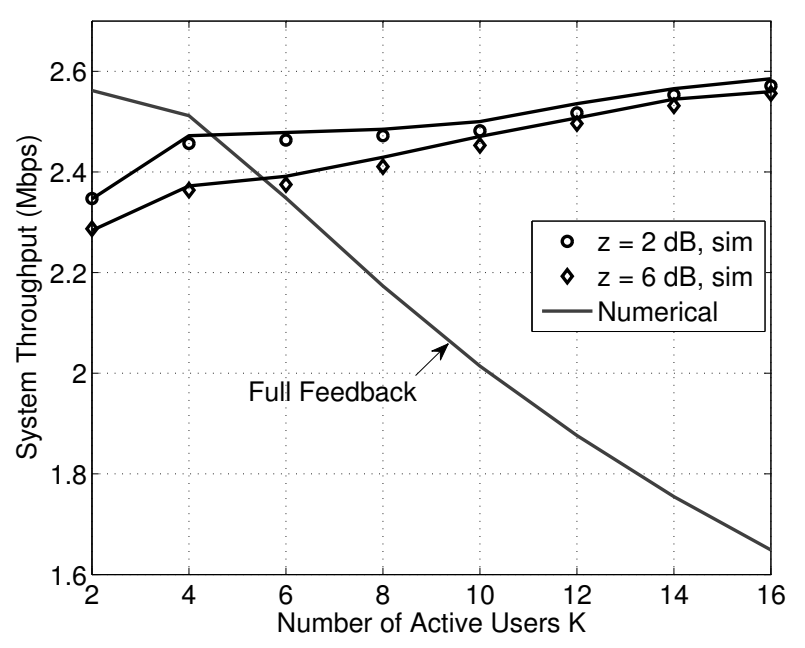

Fig. 2. Throughput performance for the proposed protocol with clustered feedback versus different number of users $(M=4)$.

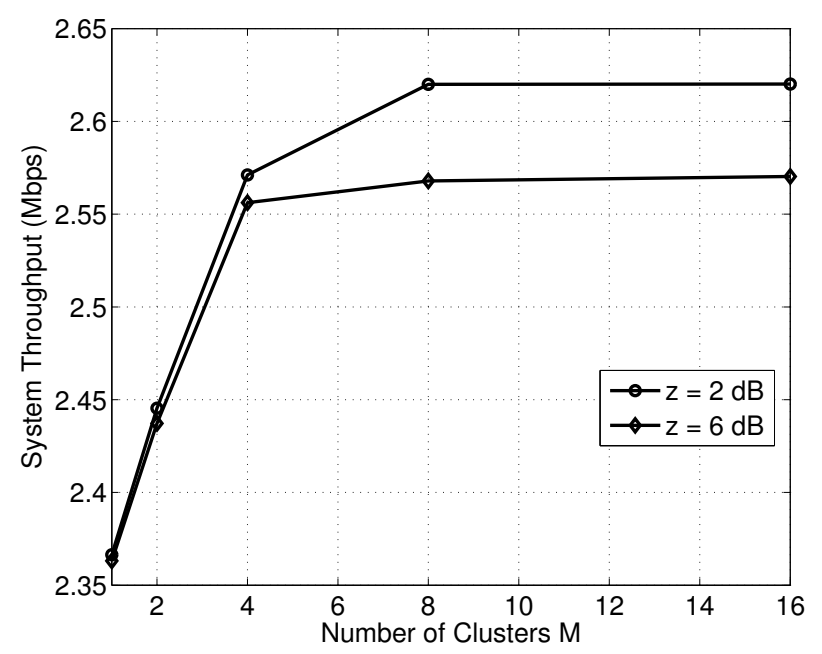

Fig. 3. Throughput performance for the proposed protocol with clustered feedback versus different number of clusters $(K=16)$.

achieves better throughput performance than the full feedback scheme, when the number of users is reasonably large.

Fig. 3 shows the throughput performance for the proposed feedback protocol versus the number of clusters, where the number of users is fixed to be 16. It can be seen from Fig. 3 that the system throughput of the proposed feedback protocol increases as the number of clusters increases. For example, there is a performance gain of about $10 \%$ in $z=2 \mathrm{~dB}$ from $M=1$ to $M=8$, where the case in $M=1$ is that of our previous protocol [10] in conventional OFDM systems.

\section{CONCLUSION}

In this paper, we have presented a cluster-based feedback protocol for multiuser OFDMA systems, where the capture effect is explicitly employed in each cluster to find the user with the best channel. We have also analyzed the throughput performance of the proposed protocol over frequency selective fading channels with UPDP. Compared with the full feedback scheme, the proposed feedback protocol reduces system overhead. Both numerical and simulation results show that the throughput performance for the proposed feedback protocol is significantly better than that of the full feedback scheme, when the number of users is reasonably large.

\section{REFERENCES}

[1] R. Knopp and P. A. Humblet, "Information capacity and power control in single-cell multiuser communications," in Proc. IEEE ICC'95, vol. 1, Seattle, Jun. 1995, pp. 331-335.

[2] D. N. C. Tse, "Optimal power allocation over parallel gaussian channels," in Proc. ISIT, Ulm, Germany, Jun. 1997, p. 27.

[3] D. Gesbert and M. Slim-Alouini, "How much feedback is multi-user diversity really worth?" in Proc. Int. Conf. on Commun., Jun. 2004, pp. 234-238

[4] C. Y. Wong, R. S. Cheng, K. B. Lataief, and R. D. Murch, "Multiuser OFDM with adaptive subcarrier, bit, and power allocation," IEEE J. Selected Areas in Commun, vol. 17, no. 10, pp. 1747-1758, Oct. 1999.

[5] Z. Shen, J. G. Andrews, and B. L. Evans, "Adaptive resource allocation in multiuser OFDM systems with proportional rate constraints," IEEE Trans. Wireless Commun, vol. 4, no. 6, pp. 2726-2737, Nov. 2005.

[6] P. Svedman, S. K. Wilson, L. J. Cimini Jr., and B. Ottersten, "Opportunistic beamforming and scheduling for OFDMA systems," IEEE Trans. Commun, vol. 55, no. 5, pp. 941-952, May. 2007.

[7] S. Sanayei and A. Nosratinia, "Opportunistic downlink transmission with limited feedback," IEEE Trans. Inf. Theory, vol. 53, no. 11, pp. 4363 4372, Nov. 2007.

[8] Somekh, O. Simeone, A.M. Haimovich, and Y. Bar-Ness, "Sum-rate analysis of general OFDM downlink channels with 1-bit feedback per subcarrier," in Proc. of CISS' 06, Mar. 2006, pp. 306-311.

[9] J. Leinonen, J. Hamalainen, and M. Juntti, "Performance analysis of downlink OFDMA frequency scheduling with limited feedback," in Proc. IEEE ICC'08, Jun. 2008, pp. 3318-3322.

[10] J. Foo and D. Huang, "Multiuser diversity with capture for wireless networks: Protocol and performance analysis," IEEE J. Select. Areas Commun, vol. 26, no. 8, pp. 1386-1396, Oct. 2008.

[11] H. Li, Q. Guo, and D. Huang, "Throughput analysis of opportunistic feedback for downlink multiuser diversity with capture effect," IEEE Commun. Lett., vol. 16, no. 1, pp. 44-46, Jan. 2012.

[12] L. Yang, M. Kang, and M.-S. Alouini, "On the capacity-fairness tradeoff in multiuser diversity systems," IEEE Trans. Veh. Technol., vol. 56, no. 4, pp. 1901-1907, Jul. 2007.

[13] C. T. Lau and C. Leung, "Capture models for mobile packet radio networks," IEEE Trans. Commun., vol. 40, no. 1, pp. 917-925, May 1992.

[14] J.-P. M. Linnartz, R. Hekmat, and R.-J. Venema, "Near-far effects in land mobile random access networks with narrow-band rayleigh fading channels," IEEE Trans. Veh. Technol, vol. 41, no. 1, pp. 77-90, Feb. 1992.

[15] T. Tang, R. W. Heath, S. Cho, and S. Yun, "Opportunistic feedback in clustered OFDM systems," in Proc. of International Symposium on Wireless personal Multimedia Communications, San Diego, CA, Sep. 2006.

[16] S. V. Amari and R. B. Misra, "Closed-form expressions for distribution of sum of exponential random variables," IEEE Trans. on Rel., vol. 46 no. 4, pp. 519-522, Dec. 1997.

[17] IEEE Standard for Local and Metropolitan Area Networks Part 16. Air Interface for Fixed Broadband Wireless Access Systems, IEEE Std. 802.16e-2005, Dec. 2005.

[18] J. So and J. M. Cioffi, "Feedback reduction scheme for downlink multiuser diversity," IEEE Trans. Wireless Commun, vol. 8, no. 2, pp. 668-671, Feb. 2009. 